\title{
Reserpine-Induced Progressive Parkinsonism in Mice Predisposed and Non-Predisposed to Depressive-Like Behavior
}

\author{
Manuela B. L. Soares', Leonardo B. Lopes-Silva', Marcela Becegato', Vinicius S. Bioni', \\ Alvaro C. Lima1, Gabriela M. Ferreira1, Ywlliane Meurer ${ }^{1,2}$, Regina H. Silva ${ }^{*}{ }^{(1)}$ \\ ${ }^{1}$ Behavioral Neuroscience Laboratory, Department of Pharmacology, Federal University of São Paulo, São Paulo, Brazil \\ ${ }^{2}$ Memory and Cognition Studies Laboratory, Post-Graduate Program of Cognitive Neuroscience and Behavior, Department of \\ Psychology, Federal University of Paraíba, João Pessoa, Brazil \\ Email: *reginahsilva@gmail.com
}

How to cite this paper: Soares, M.B.L., Lopes-Silva, L.B., Becegato, M., Bioni, V.S., Lima, A.C., Ferreira, G.M., Meurer, Y. and Silva, R.H. (2021) Reserpine-Induced Progressive Parkinsonism in Mice Predisposed and Non-Predisposed to Depressive-Like Behavior. Journal of Behavioral and Brain Science, 11, 267-279.

https://doi.org/10.4236/jbbs.2021.1111022

Received: August 13, 2021

Accepted: November 9, 2021

Published: November 12, 2021

Copyright $\odot 2021$ by author(s) and Scientific Research Publishing Inc. This work is licensed under the Creative Commons Attribution International License (CC BY 4.0).

http://creativecommons.org/licenses/by/4.0/ (c) (i) Open Access

\begin{abstract}
Parkinson's disease (PD) is a progressive degenerative condition that mainly affects the elderly. The disease comprises motor symptoms such as tremors at rest, loss of voluntary movement, decreased muscle strength, propensity to lean forward and acceleration of the walking pace. These signs are related to the degeneration of the nigrostriatal dopaminergic pathway. Patients also have non-motor symptoms, among which sleep alterations, cognitive deficits, fatigue, pain and depression stand out. Although depression has been described as the most prevalent non-motor symptom, it is not clear whether this mood disorder is due to PD or patients would already have a greater predisposition. The present study evaluated the relationship between the predisposition to depressive-like behavior and the development of motor alterations in a progressive pharmacological model of $\mathrm{PD}$ in mice. Mice were classified into groups of depressive-like propensities and submitted to the pharmacological model. Reserpine was administrated at $0.1 \mathrm{mg} / \mathrm{kg}$ on alternate days for 40 days. The catalepsy and oral movement tests were used to evaluate motor alterations, the sucrose preference test was used to evaluate anhedonia, and the open field test was applied to evaluate general activity. Reserpine promoted parkinsonian motor impairments, and there were no differences between animals from different depressive-like behavior profiles. Thus, it was not possible to find a relationship between parkinsonism and the propensity to depression based on the basal sucrose preference test. More studies with other evaluations of depressive-like behavior are needed to confirm the results found in our study.
\end{abstract}




\section{Keywords}

Parkinson's Disease, Depression, Reserpine, Mice

\section{Introduction}

Parkinson's disease (PD) is the second most prevalent neurodegenerative disease with long survival [1]. The main symptoms include bradykinesia, tremor, decreased muscle strength and postural instability [2] [3] [4]. The disease has a progressive and chronic profile, and aging is the main risk factor [5]. The diagnosis is mainly based on the motor symptoms (MS). However, by the time the MS begin, approximately $60 \%$ of the neurons in the substantia nigra pars compacta (SNpc) and $80 \%$ of the neurons in the striatum are already lost [6]. In addition, other areas of the brain can also be affected by neurodegeneration, giving rise to the prodromal stage. This stage is characterized by the emergence of the non-motor symptoms (NMS) such as sleep alterations, fatigue, apathy, cognitive deficits, pain, autonomic disorders and depression [7]. Those symptoms have recently been recognized as important components of the disease, and may be useful for early diagnosis [8] [9] [10]. In addition, the effective treatment of NMS is extremely important, as it has a high impact on the patient's quality of life [11].

Depression is the most prevalent NMS in PD, being present in at least $25 \%$ of cases [7] [12], reaching up to $90 \%$ [13]. It is present regardless of age, but there is a significant increase of prevalence as disease severity progresses [14].

PD patients with depression have a less favorable prognosis when compared with those without depressive symptoms, as they tend to perform worse on motor assessment scales and in daily activities. They also present more cognitive symptoms and poorer quality of life. Despite this, the diagnosis is still flawed, resulting in inadequate treatment or even no treatment for the depressive condition [13]. Nevertheless, there is a good response when correct treatment is carried out [15].

Julien and colleagues [16] proposed that the individual with PD tends to move away from social situations in order to reduce stress and anxiety related to the motor symptoms. Thus, it is possible that vulnerability to depression increases because of social isolation and reduction of rewarding interactions [16]. These factors increase the patient's degree of suffering, further compromising their independence [15].

In addition, the presence of other NMS such as dementia, psychotic episodes, anxiety and sleep disorders have been associated with an increased risk for depression. In summary, depression in PD may be related to one of three factors (or a combination of them): a consequence of the pathology of PD (decrease in the release of catecholamines); a reaction to the disability associated with PD (stress caused by motor symptoms and/or social isolation and/or other NMS); or 
an isolated phenomenon unrelated to the pathology [7] [17].

The purpose of this study was to investigate a possible relationship between the predisposition to depressive-like behavior and motor alterations in mice submitted to progressive parkinsonism induced by repeated administration of a low dose of reserpine. Reserpine is a blocker of the vesicular monoamine transporter and the chronic low-dose treatment with this drug has shown progressive motor, non-motor and neuronal alterations compatible with PD pathology [18] [19].

\section{Materials and Methods}

\section{Animals}

Five-month-old male Swiss mice were obtained from the Central Bioterium of the Federal University of São Paulo (CEDEME/UNIFESP). They were kept in a maximum of 5 animals per plastic cage $(30 \times 20 \times 12 \mathrm{~cm})$, under controlled ventilation and temperature $\left(22^{\circ} \mathrm{C} \pm 1{ }^{\circ} \mathrm{C}\right)$, light/dark cycle of $12 / 12 \mathrm{~h}$ with lights on 6:30 a.m., with free access to water and food. The local Ethics Committee (CEUA/UNIFESP) approved all procedures. All efforts were made to minimize animal pain, suffering or discomfort.

\section{Experimental design and drug treatment}

Forty-eight mice were submitted to Sucrose Preference Test (SPT) before the beginning of the experiments in order to rank them based on the anhedonia profile. We considered the anhedonic behavior as a depressive-like trait. The animals were divided into three groups of sixteen animals each: the ones with greater depressive-like behavior (more pronounced anhedonic behavior in the SPT, predisposed group), the ones with less depressive-like behavior (non-predisposed group) and the ones with intermediate scores. The intermediate group was not included in the experiment in order to ensure that the subjects included in the study were categorized in the two extremes of the depressive-like behavior spectrum. Mice of the predisposed and the non-predisposed groups were randomly distributed into two subgroups: reserpine and vehicle.

Reserpine (Sigma Chemical Co. St Louis, MO) was dissolved in glacial acetic acid and diluted to the correct concentration in distilled water $(0.1 \mathrm{mg} / \mathrm{kg})$. Vehicle consisted of the same amount of acetic acid and distilled water as in the reserpine solution. These solutions were injected by subcutaneous route (s.c.) at a volume of $10 \mathrm{~mL} / \mathrm{kg}$ of body weight.

Twenty injections of vehicle or reserpine were administrated on alternate days for forty days. During this period, tests were performed to evaluate the motor function of the animal-catalepsy and oral movement (OM) tests and to evaluate the depressive-live behavior-SPT-as illustrated in Figure 1. At the end of the experiment, all animals were euthanized.

\section{Sucrose Preference Test (SPT)}

During the adaptation phase, the animals were placed in individual cages with access to two bottles of water. After 24 hours, one of the bottles was replaced by 


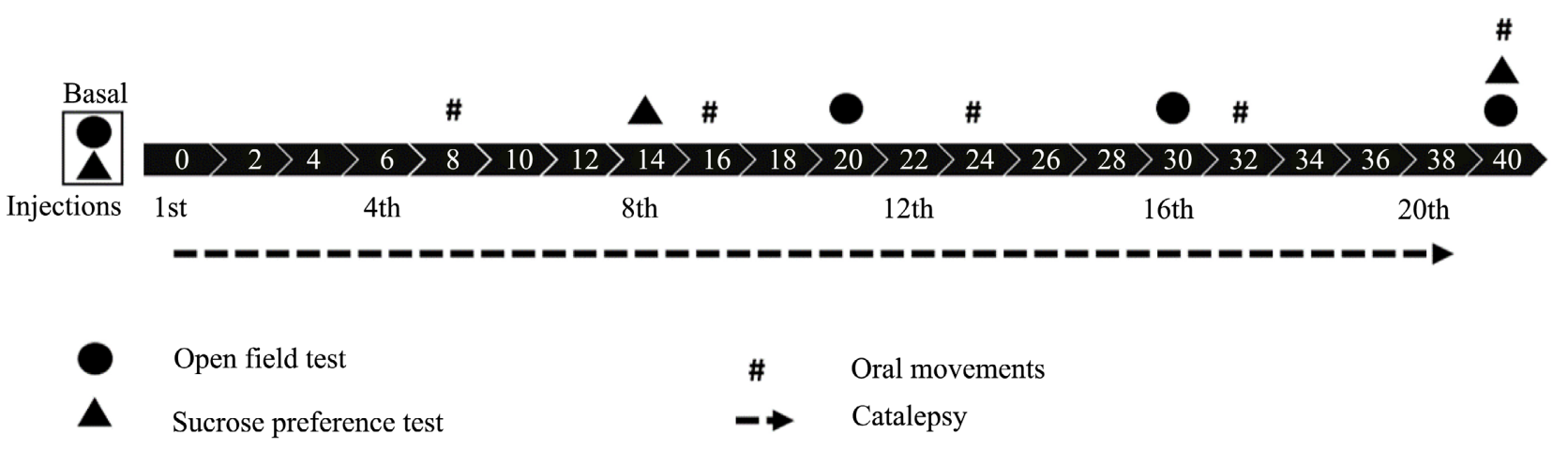

Figure 1. Schematic representation of the experimental design.

another containing $0.8 \%$ sucrose solution, which remained in the individual cage for another 24 hours. The contents of both bottles were weighted before and after the experiment period to quantify consumption. The rate of sucrose preference (sucrose consumption-water consumption/total consumption) was evaluated.

\section{Open Field}

The open field test was held on a circular arena made of wood with an opaque white polyethylene cylindrical wall $(40 \mathrm{~cm}$ diameter base and $50 \mathrm{~cm}$ high wall). The test lasted five minutes. Each animal was placed in the central region of the apparatus and individually evaluated. The parameters registered were the total distance traveled and the duration of grooming and immobility.

\section{Catalepsy}

This test was conducted by placing the animal with both forepaws on a horizontal glass bar positioned $5 \mathrm{~cm}$ above the surface of the bench supporting the hind paws. The time each animal remained in this position until removing at least one of the forepaws from the bar was measured up to a maximum 180 seconds. Each animal was tested three consecutive times and the three measurements were averaged.

\section{Oral Movements}

Parameters related to oral dyskinesia were measured by placing the animals individually in barred cages $(29 \times 21 \times 24 \mathrm{~cm})$, where the animals were observed for 10 minutes. Mirrors were fixed under and behind the cage, allowing the observer/experimenter to see the animal in any location. The number of chewing movements that were not directed to any object (vacuous chewing movements) was registered.

\section{Statistical Analysis}

The statistical analysis was conducted with the IBM-SPSS Statistics software, version 20.0. The data were analyzed by using two-way repeated measures analysis of variance (ANOVA), depending on the experimental procedure. Posterior analyses were performed by Sidak post-hoc. ANOVA with repeated measures was used for evaluations over time. The Student's $\mathrm{T}$ test was used in the analysis of the basal evaluation of the sucrose preference test. Differences were consi- 
dered statistically significant when $\mathrm{p}<0.05$.

\section{Results}

\section{Basal Sucrose Preference}

Non-predisposed animals presented greater preference for sucrose $[\mathrm{T}(32)=$ -8.751; $\mathrm{p}<0.05$ ] (Figure 2). This difference was expected, as this was the parameter used to categorize the predisposition groups.

\section{Catalepsy test}

Chronic administration with reserpine at a low dose $(0.1 \mathrm{mg} / \mathrm{kg})$ induced progressive increase in the duration of catalepsy behavior. The observation days of the catalepsy test were compiled into 4 phases, each corresponding to 10 days (or 5 reserpine or vehicle injections).

ANOVA with repeated measures (phases) and treatment and predisposition as between subject factors, followed by Sidak's post-hoc, indicated that there was a significant difference between treated and non-treated groups beginning at the third phase of treatment (after the 10th injection of reserpine) $[\mathrm{F}(3,87)=9.185$; $\mathrm{p}<0.05$ ]. No statistical differences were observed between predisposed and non-predisposed groups within the same treatment (Figure 3).

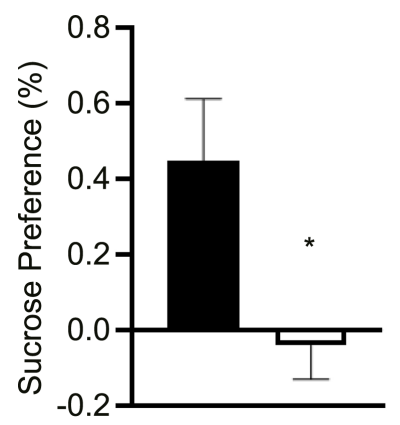

Non-predisposed

$\square$ Predisposed

Figure 2. Basal evaluation of sucrose preference. Mean + S.D.M. of percent of sucrose solution consumption. ${ }^{*} \mathrm{p}<0.05$ predisposed group $(\mathrm{n}=16)$ compared to the non-predisposed group $(\mathrm{n}=16)$ (Student's T test).

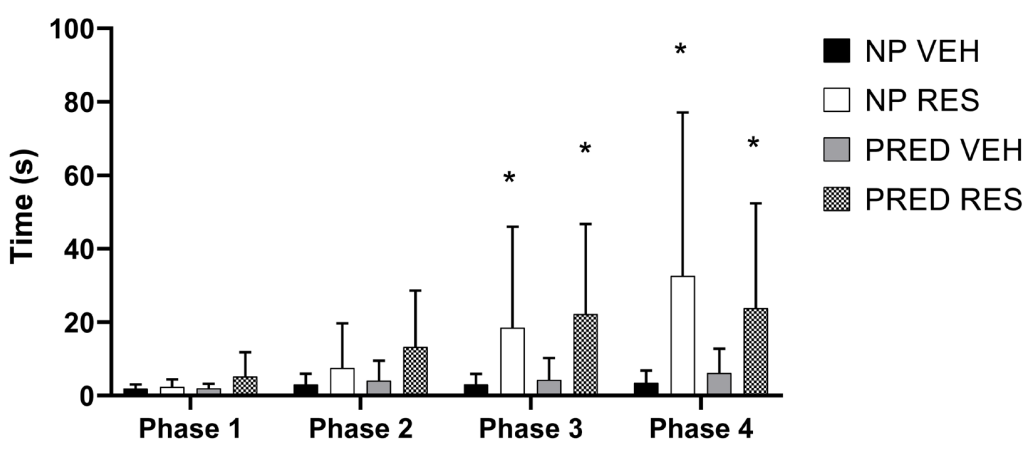

Figure 3. Effect of treatment with reserpine on duration of catalepsy(s) throughout the phases of treatment, expressed as mean + S.D.M. Groups $(n=8)$ were divided according to predisposition to depressive-like behavior (NP = non-predisposed; PRED = predisposed) and treatment $(\mathrm{VEH}=$ vehicle; RES $=$ reserpine $) .{ }^{*} \mathrm{p}<0.05$ effect of reserpine treatment (ANOVA with repeated measures and Sidak post-hoc). 


\section{Open field}

The open-field test was performed at four time points across the protocol: before the beginning of treatment (basal), and after the 10th, 15th, and 20th injections. The total distance traveled by the animal within the arena during the five minutes period was recorded to assess motor activity. ANOVA with repeated measures (sessions) and treatment and predisposition as between subject factors, followed by Sidak post-hoc, indicated that there was a significant difference only after the 20th injection. The effect of reserpine was detected within the nonpredisposed subjects, i.e., non-predisposed treated animals presented decreased locomotion compared to the non-predisposed untreated mice $[\mathrm{F}(3,32)=4.398$; $\mathrm{p}<0.05$ ] (Figure 4).

The analyses of the other parameters evaluated in the open field test did not yield significant differences (immobility duration: $\mathrm{F}(3,81)=6.073 ; \mathrm{p}>0.05$ and grooming duration: $\mathrm{F}(3,81)=6.751 ; \mathrm{p}<0.05$; data not shown).

\section{Oral Movements}

The oral movements test was performed five times during the treatment period: after the 4th, 8th, 12th, 16th and 20th injections. ANOVA with repeated measures (sessions) and treatment and predisposition as between subject factors followed by Sidak post-hoc found significant difference between treated and non-treated animals in the observations conducted after the 8th, 12th and 20th injections $[F(4,116)=8.681 ; p<0.05]$. There was an increase in vacuous chewing movements in treated animals, but no effect of predisposition (Figure 5).

\section{Sucrose Preference Test}

The SPT was performed at three-time points throughout the treatment: at baseline and after the 7th and 20th injections. As previously mentioned, because this was the test of choice for categorizing the predisposition groups, a statistical difference was observed between predisposed and non-predisposes animals in the baseline period (as described previously, item 3.1). However, this effect did not endure across treatment $[F(2,58)=0.972 ; p>0.05]$. ANOVA with repeated measures did not reveal any effect of the treatment, nor of the treatment $\mathrm{x}$ time interaction (Figure 6).

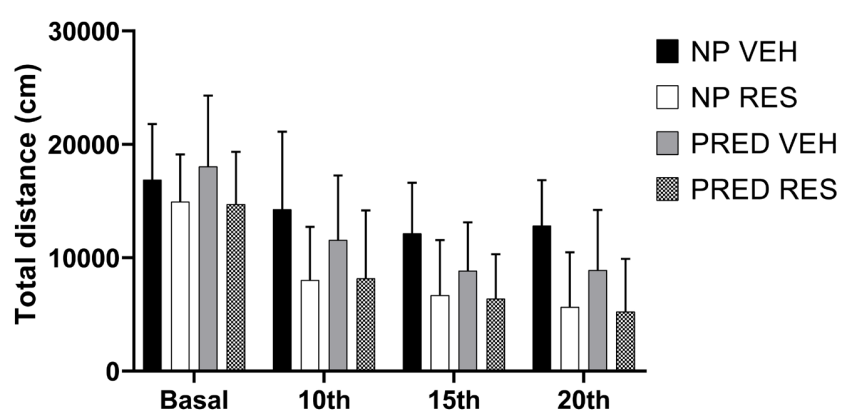

Figure 4. Effect of treatment and predisposition on total distance traveled in the open field over time expressed as mean + S.D.M. Groups $(n=8)$ were divided according to predisposition to depressive-like behavior $(\mathrm{NP}=$ non-predisposed; $\mathrm{PRED}=$ predisposed $)$ and treatment $(\mathrm{VEH}=$ vehicle; $\mathrm{RES}=$ reserpine $){ }^{*} \mathrm{p}<0.05$ effect of reserpine treatment (ANOVA with repeated measures and Sidak post-hoc). 


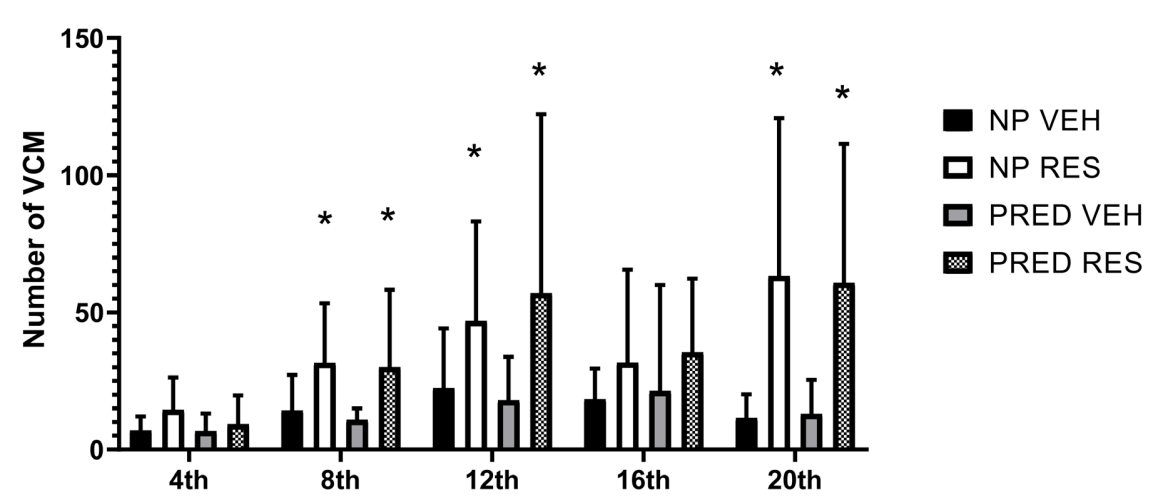

Figure 5. Number of vacuous chewing movements (VCM) in the oral movement test throughout the treatment expressed in mean + S.D.M. Groups $(n=8)$ were divided according to predisposition to depressive-like behavior (NP = non-predisposed; PRED = predisposed $)$ and treatment $(\mathrm{VEH}=$ vehicle; RES $=$ reserpine $){ }^{\star} \mathrm{p}<0.05$ effect of reserpine treatment (ANOVA with repeated measures and Sidak post-hoc).

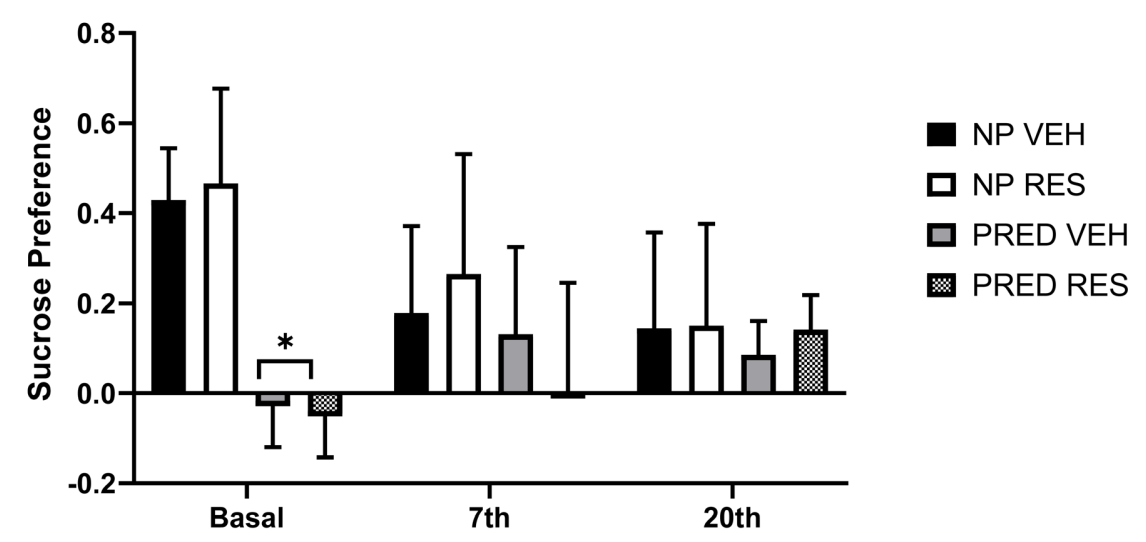

Figure 6. Sucrose preference throughout treatment expressed as mean + S.D.M. Groups $(\mathrm{n}=8)$ were divided according to predisposition to depressive-like behavior (NP = non-predisposed; PRED = predisposed $)$ and treatment $(\mathrm{VEH}=$ vehicle; $\mathrm{RES}=$ reserpine $)$ ${ }^{*} \mathrm{p}<0.05$ compared to NP groups (ANOVA with repeated measures and Sidak post-hoc).

\section{Discussion}

In the present study, we performed the chronic administration of reserpine at $0.1 \mathrm{mg} / \mathrm{kg}$ (considered a low dose) on alternate days for 40 days. This protocol has proved to be effective in inducing progressive parkinsonism in mice, increasing catalepsy duration. Previous studies with rodents (both rats and mice) have demonstrated a similar effect, showing that treatment with reserpine promoted motor impairment in a gradual manner, allowing the analysis of the pre-motor period of the disease [18] [20] [21]. It is worth noting that although this protocol was first preconized in rats [18] [21], the progressive profile of catalepsy was also demonstrated in mice [22]. This treatment protocol was chosen because acute or sub chronical high doses of reserpine $(1.0-5.0 \mathrm{mg} / \mathrm{kg})$ usually cause severe motor damage, precluding the evaluation of non-motor alterations [23]. A similar pattern is observed in other pharmacological models such as 6-hydroxydopamine (6-OHDA) and MTPT, which fail to reproduce early signs 
of PD due to the high toxicity to dopamine neurons [24] [25] [26].

In this study, using the chronic low-dose reserpine protocol, we observed the onset of motor symptoms in phase 3 of treatment, i.e., from the 10th injection onwards. This increase in catalepsy corroborates previous findings [18] [21] [22]. We also observed an increase in the number of vacuous chewing movements after the 8th, 12th and 20th injections, consistent with previous similar protocols [20] [22]. Finally, the results showed that the onset of motor symptoms in subjects with greater predisposition to depressive-like behavior did not differ from the non-predisposed group. Indeed, both groups displayed the onset of motor impairment in the same period (phase 3 ).

The open field test assesses several behavioral parameters, including the animal's motor integrity based on locomotion within the arena. Previous studies have shown that treatment with reserpine did not result in motor impairment in the open field after the 4th injection [21], but resulted in reduction of locomotion after 10 injections in rats [20] or 15 injections in mice [22]. Herein, this impairment was found only in the non-predisposed animals, i.e. the animals treated with reserpine showed a decrease in total locomotion after the 20th injection. Unlike the catalepsy test, which evaluates almost exclusively motor function, the open field test encompasses a more global evaluation of the animal's general activity, including motivational and cognitive aspects of exploratory behavior. This difference may explain the absence/minimization of the effects of reserpine treatment on the open field test compared to the results of the catalepsy test.

Reserpine leads to parkinsonian symptoms by blocking the vesicular monoamine transporter (VMAT2) in the central nervous system, depleting the vesicular content and increasing the level of monoamines, including dopamine, in the pre-synaptic cytosol. The accumulation of dopamine leads to increased degradation of this neurotransmitter by the enzyme monoamine oxidase (MAO), generating reactive oxygen species (ROS) and reactive quinones (DOPAC-Q), which results in increased oxidative stress (for review see [19]).

As mentioned, the primary cause of $\mathrm{PD}$ is the decrease in dopamine release in the striatum. However, there are also impairments of the noradrenergic, serotonergic and cholinergic systems, which result in the appearance of non-motor symptoms, including depression and cognitive impairments [27] [28] [29]. In addition, the dopaminergic mesolimbic pathway is part of the reward and motivation system. There is evidence that decreased dopamine in this pathway contributes to the pathophysiology of depression [30] [31]. This fact would explain why conventional antidepressants that act on serotonin and norepinephrine-such as SSRI and tricyclics-are not always able to improve some specific symptoms, like anhedonia and decreased motivation [31] [32].

In animal models, anhedonia can be evaluated by the SPT. In this test, animals with the lowest rate of sucrose consumption are considered less motivated when compared to those with a higher rate. Studies have shown that the administration of 6-OHDA resulted in a decrease in the sucrose preference when compared 
to control, and this behavior could be related to dysfunction of the dopaminergic and noradrenergic pathways of the limbic reward system [33].

The present study showed that the low-dose reserpine treatment did not result in a decrease in the SPT, indicating that under this protocol the motivational behavior does not change, despite the fact that reserpine is known to promote depletion of monoamines. Previous studies have shown that treatment with reserpine at a dose of $2 \mathrm{mg} / \mathrm{kg}$ (above the dose used in the present study) was able to reduce the rate of sucrose preference in animal models, while the dose of 1 $\mathrm{mg} / \mathrm{kg}$ did not promote this effect [34], suggesting that this effect is dose dependent.

Reserpine was initially used as an antihypertensive drug [35]. However, the clinical use of the drug was subsequently restricted because it also causes parkinsonian and depressive symptoms [36]. More recently, Zhu and colleagues [37] conducted a translational study investigating the association between low doses of reserpine and the incidence of depression in hypertensive patients. As a result, the study did not find significant differences in the prevalence of depression among reserpine users and controls. In this respect, Baumeister and colleagues [38] have shown that most individuals treated with reserpine for hypertension already had pre-existing mental disorders (including depression), suggesting that there was a higher probability of depression in these cases than in the general population. In the present study, as mentioned, there was no evidence that reserpine induced depressive-like behavior in the animals, at least under our protocol conditions (repeated low dose). Conversely, Campêlo and colleagues [22] demonstrated a decrease in BDNF (brain derived neurotrophic factor) levels in animals chronically treated with reserpine, in a protocol similar to the one applied here. That effect was reversed when the animals were submitted to environmental enrichment, a procedure known to be protective against stressful stimuli by regulating glucocorticoid receptors. In this respect, decreased levels of BDNF in depressed individuals have been reported, suggesting that this factor is related to the pathophysiology of depression [31]. Nevertheless, Campêlo and colleagues [22] did not evaluate depressive-like behavior in that study. Taken together, the evidences suggest that the effect of reserpine on depression is dependent on protocol of treatment, as well as related to the predisposition to the disease [38].

Despite the reserpine-induced motor impairment was consistent with previous studies [18] [20] [21], we did not observe reserpine effects on the depressive-like behavior, which differs from studies conducted previously both in animal models [34] and in human trials [38] [39]. This difference may be related to methodological differences. Indeed, this work conducted a previous assessment of the predisposition to the depressive-like behavior, while previous studies included only post-treatment data.

Finally, it is important to mention that the use of only one parameter (anhedonia) to categorize the animals according to their propensity to depression is a limitation of the study. Indeed, depression comprises a myriad of signs, and SPT 
assessment of anhedonia alone does not convey the complexity of the condition. Thus, the present study is an initial step to the investigation of depression predisposition as a risk factor for PD in animal models, but the enlargement of studies approaching other depression-like behaviors is desirable.

\section{Conclusion}

The present study was not able to show a link between predisposition to depressive-like behavior and the motor alterations related to PD. However, as only SPT behavior was taken into account to evaluate depression propensity, studies with other evaluations of depressive-like behavior are needed to confirm the results found in our study. As mentioned in the introduction, although several epidemiologic studies show a strong prevalence of depression in DP patients, there is no definitive answer to whether depression in $\mathrm{PD}$ is originated from the pathology itself or if there is a co-predisposition between the conditions. Further studies are needed to understand the pathophysiology of both diseases and what are their common grounds, which could explain why depression is the most common non-motor symptom in $\mathrm{PD}$.

\section{Acknowledgements}

This study was financed by Fundação de Amparo à Pesquisa do estado de São Paulo-FAPESP (grant 2017/26253-3), and by Coordenação de Aperfeiçoamento de Pessoal de Nível Superior/Brasil-CAPES (finance code 001). RHS is a recipient of a research fellowship from Conselho Nacional de Desenvolvimento Científico e Tecnológico (CNPq, grant 303325/2017-8). The authors would like to thank Claudenice Moreira dos Santos for capable technical assistance.

\section{Conflicts of Interest}

The authors declare no conflicts of interest regarding the publication of this paper.

\section{References}

[1] Ascherio, A. and Schwarzschild, M.A. (2016) The Epidemiology of Parkinson's Disease: Risk Factors and Revention. The Lancet Neurology, 15, 1257-1272. https://doi.org/10.1016/S1474-4422(16)30230-7

[2] Berrios, G.E. (2016) Introdução à "Paralisia agitante", de James Parkinson (1817). Revista Latinoamericana de Psicopatologia Fundamental, 19, 114-121. https://doi.org/10.1590/1415-4714.2016v19n1p114.9

[3] Postuma, R.B., Berg, D., Stern, M., Poewe, W., Olanow, W., Oertel, W., Obeso, J., Marek, K., Litvan, I., Lang, A.E., Halliday, G., Goetz, C.G., Gasser, T., Dubois, B., Chan, P., Bloem, B.R., Adler, C.H. and Deuschi, G. (2015) MDS Clinical Diagnostic Criteria for Parkinson's Disease. Movement Disorders, 39, 1591-1599. https://doi.org/10.1002/mds.26424

[4] Lotharius, J. and Brundin, P. (2002) Pathogenesis of Parkinson's Disease: Dopamine, Vesicles and Alpha-Synuclein. Nature Reviews Neuroscience, 3, 932-942. https://doi.org/10.1038/nrn983 
[5] Steidl, E.M.S., Ziegler, J.R. and Ferreira, F.V. (2007) Doença de Parkinson: Revisão Bibliográfica. Disciplinarum Scientia, 8, 115-129.

[6] Cheng, H.C., Ulane, C.M. and Bruke, R.E. (2010) Clinical Progression in Parkinson's Disease and the Neurobiology of Axons. Annals of Neurology, 67, 715-725. https://doi.org/10.1002/ana.21995

[7] Schapira, A.H.V., Chaudhuri, K.R. and Jenner, P. (2017) Non-Motor Features of Parkinson Disease. Nature Reviews Neuroscience, 18, 435-450. https://doi.org/10.1038/nrn.2017.62

[8] Pagonabarraga, J., Kulisevsky, J., Strafella, A.P. and Krack, P. (2015) Apathy in Parkinson's Disease: Clinical Features, Neural Substrates, Diagnosis, and Treatment. The Lancet Neurology, 14, 528-531. https://doi.org/10.1016/S1474-4422(15)00019-8

[9] Pont-Sunyer, C., Hotter, A., Gaig, C., Seppi, K., Compta, Y., Katzenschlager, R., Mas, N., Hofeneder, D., Brücke, T., Bayés, A., Enzel, K., Infante, J., Zach, H., Pirker, W., Posada, I.J., Álvarez, R., Ispierto, L., De Fàbregues, O., Callén, A., Palasó, A., Aguilar, M., Martí, M.J., Vallderiola, F., Salamero, M., Poewe, W. and Tolosa, E. (2014) The Onset of Nonmotor Symptoms in Parkinson's Disease (The ONSET PD Study). Movement Disorders, 30, 229-237. https://doi.org/10.1002/mds.26077

[10] Martinez-Martin, P., Chaudhuri, K.R., Rojo-Abuin, J.M., Rodriguez-Blazquez, C., Alvarez-Sanchez, M., Arakaki, T., Bergareche-Yarza, A., Chade, A., Garreto, N., Gershanik, O., Kurtis, M.M., Martinez-Castrillo, J.C., Mendoza-Rodriguez, A., Moore, H.P., Rodriguez-Violante, M., Singer, C., Tilley, B.C., Huang, J., Stebbins, G.T. and Goetz, C.G. (2013) Assessing the Non-Motor Symptoms of Parkinson's Disease: MDS-UPDRS and NMS Scale. European Journal of Neurology, 22, 37-43. https://doi.org/10.1111/ene.12165

[11] Draoui, A., El Hiba, O., Aimrane, A., El Khiat, A. and Gamrani, H. (2020) Parkinson's Disease: From Bench to Bedside. Revue Neurologique, 176, 543-559. https://doi.org/10.1016/j.neurol.2019.11.002

[12] Cumming, J.L. (1992) Depression and Parkinson's Disease: A Review. The American Journal of Psychiatry, 149, 443-454. https://doi.org/10.1176/ajp.149.4.443

[13] Reijnders, J.S.A.M., Ehrt, U., Weber, W.E.J., Aarsland, D. and Leentjens, A.F.G. (2008) A Systematic Review of Prevalence Studies of Depression in Parkinson's Disease. Movement Disorders, 23, 183-189. https://doi.org/10.1002/mds.21803

[14] Riedel, O., Heuser, I., Klotsche, J., Dodel, R. and Wittchen, H.U. (2010) Occurrence Risk and Structure of Depression in Parkinson Disease with and without Dementia: Results from GEPAD Study. Journal of Geriatric Psychiatry and Neurology, 23, 27-34. https://doi.org/10.1177/0891988709351833

[15] Nilsson, F.M., Kessing, L.V. Sørensen, T.M., Andersen, P.K. and Bolwig, T.G. (2002) Major Depressive Disorder in Parkinson's Disease: A Register-Based Study. Acta Psychiatrica Scandinavica, 106, 202-211. https://doi.org/10.1034/j.1600-0447.2002.02229.x

[16] Julien, C.L., Rimes, K.A. and Brown, R.G. (2016) Rumination and Behavioural Factors in Parkinson's Disease Depression. Journal of Psychosomatic Research, 82, 48-52. https://doi.org/10.1016/j.jpsychores.2016.01.008

[17] Grover, S., Somaiya, N., Kumar, S. and Avasthi, A. (2015) Psychiatric Aspects of Parkinson's Disease. Journal of Neurosciences in Rural Practice, 6, 65-76. https://doi.org/10.4103/0976-3147.143197

[18] Santos, J.R., Cunha, J.A.S., Dierschnabel, A.L., Campêlo, C.L.C., Leão, A.H.F.F., Silva, A.F., Engelberth, R.C.G.J., Izídio, G.S., Cavalcante, J.S., Abílio, V.C., Ribeiro, A.M. and Silva, R.H. (2013) Cognitive, Motor and Tyrosine Hydroxylase Temporal 
Impairment in a Model of Parkinsionism Induced by Reserpine. Behavioural Brain Research, 253, 68-77. https://doi.org/10.1016/j.bbr.2013.06.031

[19] Leão, A.H., Sarmento-Silva, A.J. and Santos, J.R. (2015) Molecular, Neurochemical, and Behavioral Hallmarkers of Reserpine as a Model for Parkinson's Disease: New Perspectives to a Long-Standing Model. Brain Pathology, 25, 377-390.

https://doi.org/10.1111/bpa.12253

[20] Leão, A.H.F.F., Meurer, Y.S.R., da Silva, A.F., Medeiros, A.M., Campêlo, C.L.C., Abílio, V.C., Engelberth, R.C.G.K., Cavalvante, J.S., Izídio, G.S., Ribeiro, A.M. and Silva, R.H. (2017) Spontaneously Hypertensive Rats (SHR) Are Resistant to a Reserpine-Induced Progressive Model of Parkinson's Disease: Differences in Motor Behavior, Tyrosine Hydroxylase and $\alpha$-Synuclein Expression. Frontiers in Aging Neuroscience, 9, Article No. 78.

[21] Fernandes, V.S., Santos, J.R., Leão, A.H., Medeiros, A.M., Melo, T.G., Izídio, G.S., Cabral, A., Ribeiro, R.A., Abílio, V.C., Ribeiro, A.M. and Silva, R.H. (2012) Repeated Treatment with a Low Dose of Reserpine as a Progressive Model of Parkinson's Disease. Behavior Brain Research, 231, 154-163.

https://doi.org/10.1016/j.bbr.2012.03.008

[22] Campêlo, C.L.C., Santos, J.R., Silva, A.F., Dierschnabel, A.L., Pontes, A., Cavalcante, J.S., Ribeiro, A.M. and Silva, R.H. (2017) Exposure to an Enriched Environment Facilitates Motor Recovery and Prevents Short-Term Memory Impairment and Reduction of Stiatal BDNF in a Progressive Pharmacological Model of Parkinsonism in Mice. Behavior Brain Research, 328, 138-148.

https://doi.org/10.1016/j.bbr.2017.04.028

[23] Grandi, L.C., Di Giovanni, G. and Galati, A. (2018) Animal Models of Early-Stage Parkinson's Disease and Acute Dopamine Deficiency to Study Compensatory Neurodegenerative Mechanisms. Journal of Neuroscience Methods, 308, 205-218. https://doi.org/10.1016/j.jneumeth.2018.08.012

[24] Deumens, R., Blokland, A. and Prickaerts, J. (2002) Modeling Parkinson's Disease in Rats: An Evaluation of 6-OHDA Lesions of the Nigrostriatal Pathway. Experimental Neurology, 175, 303-317.

[25] Schober, A. (2004) Classic Toxin-Induced Animal Models of Parkinson's Disease: 6-OHDA and MPTP. Cell Tissue Res, 318, 215-224.

[26] Jiang, P.E., Lang, Q.H., Yu, Q.Y., Tang, X.Y., Liu, Q.Q., Li, X.Y. and Feng, X.Z. (2019) Behavioral Assessments of Spontaneous Locomotion in a Murine MPTPInduced Parkinson's Disease Model. Journal of Visualized Experiments, 7. https://doi.org/10.3791/58653

[27] Han, Q.W., Yuan, Y.H. and Chen N.H. (2019) The Therapeutic Role of Cannabinoid Receptors and Its Agonists or Antagonists in Parkinson's Disease. Progress in Neuro-Psychopharmacology \& Biological Psychiatry, 96, Article ID: 109745. https://doi.org/10.1016/j.pnpbp.2019.109745

[28] Kaji, Y. and Hirata, K. (2011) Apathy and Anhedonia in Parkinson's Disease. International Scholarly Research Notices, 2011, Article ID: 219427. https://doi.org/10.5402/2011/219427

[29] Remy, P., Doder, M., Lees, A., Turjanski, N. and Brooks, D. (2005) Depression in Parkinson's Disease: Loss of Dopamine and Noradrenaline Innervation in the Limbic System. Brain, 128, 1324-1322. https://doi.org/10.1093/brain/awh445

[30] Cléry-Melin, M., Jollant F. and Gorwood, P. (2018) Reward Systems and Cognitions in Major Depressive Disorder. CNS Spectrums, 24, 64-77.

https://doi.org/10.1017/S1092852918001335 
[31] Dean, J. and Keshavan, M. (2017) The Neurobiology of Depression: An Integrated View. Asian Journal of Psychiatry, 27, 101-111. https://doi.org/10.1016/j.ajp.2017.01.025

[32] Belujon, P. and Grace, A.A. (2017) Dopamine System Dysregulation in Major Depressive Disorders. International Journal of Neuropsychopharmacology, 20, 1036-1046. https://doi.org/10.1093/ijnp/pyx056

[33] Kaminska, K., Lenda, T., Konieczny, J., Czarnecka, A. and Lorenc-Koci, E. (2017) Depressive-Like Neurochemical and Behavioral Markers of Parkinson's Disease after 6-OHDA Administered Unilaterally to the Rat Medial Forebrain Bundle. Pharmacological Reports, 69, 985-994. https://doi.org/10.1016/j.pharep.2017.05.016

[34] Skalisz, L.L., Beijamini, V., Joca, S.L., Vital, M.A.B.F., Da Cunha, C. and Andreatini, R. (2002) Evaluation of the Face Validity of Reserpine Administration as an Animal Model of Depression-Parkinson's Disease Association. Progress in Neuro-Psychopharmacology \& Biological Psychiatry, 26, 879-883.

https://doi.org/10.1016/S0278-5846(01)00333-5

[35] Mashour, N.H., Lin, G.I. and Frishman, W.H (1998) Herbal Medicine for the Treatment of Cardiovascular Disease: Clinical Considerations. Archives of Internal Medicine, 158, 2225-2234. https://doi.org/10.1001/archinte.158.20.2225

[36] Rejintes, M. and Meyer, P.T. (2019) No Free Lunch with Herbal Preparations: Lessons from a Case of Parkinsonism and Depression Due to Herbal Medicine Containing Reserpine. Frontiers in Neurology, 23, 183-189. https://doi.org/10.3389/fneur.2019.00634

[37] Zhu, G.H., Sun, X.P., Li, J., Pi, L., Tang, H.Q., Gao, H.Q., Cong, H.L., Qu, P., Lu, X.Z., Zhang, X.J., Zhao, L.S., Gui, Y.F., Liu, D.X., Zhang, L.Q., Tang, H., Hu, Y.X., Fan, L. and Hua, Q. (2019) No Association between Low-Dose Reserpine Use and Depression in Older Hypertensive Patient: Result of a Multicenter, Cross-Sectional Study. Journal of Geriatric Cardiology, 16, 608-613.

[38] Baumeister, A.A., Hawkins, M.F. and Uzelac, S.M. (2003) The Myth of Reserpine-Induced Depression: Role in the Historical Development of the Monoamine Hypothesis. Journal of the History of the Neurosciences, 12, 207-220. https://doi.org/10.1076/jhin.12.2.207.15535

[39] Lemieux, G., Davignon, A. and Genest, J. (1956) Depressive States during Rauwolfia Therapy for Arterial Hypertension: A Report of 30 Cases. Canadian Medical Association Journal, 74, 522-526. 